gar Wagner. „Die Daten werden für Zwecke der Kundenbindung, der Online-Werbung oder der Marktforschung erfasst und ausgewertet und zu individuellen Nutzungs-, Kauf- oder Bewegungsprofilen verdichtet. Je mehr das Internet im Alltag genutzt wird, desto mehr Datenspuren liefern Hinweise auf Interessen, Vorlieben und Verhaltensweisen der Nutzerinnen und Nutzer. Den digitalen Augen und Ohren im Internet nicht alles preiszugeben, ist das legitime Recht aller Nutzer", so Wagner. "Die Globalität des Internet macht es häufig jedoch schwer, dies einzufordern. Wer nicht will, dass seine Daten Neugier und Sammelwut preisgegeben sind, sollte digitale Vorsorge treffen" so der Datenschutzbeauftragte.

Zwar bringe es die digitale Lebenswelt mit sich, dass man nicht immer und vollständig anonym bleiben könne; darin gleiche sie letztlich dem analogen Alltag. Hier wie dort wechsele man zwischen notwendiger Preisgabe persönlicher Daten und berechtigtem Verschweigen.

Welche Möglichkeiten bestehen, Datenspuren im Internet zu vermeiden, wie Inhalte bei E-Mail und Online-Speichern durch Verschlüsselung geschützt werden können oder wie die penetrante Dateninkontinenz von Smartphones unterbunden werden kann, erläutert der Landesbeauftragte für den Datenschutz auf seinem Internet-Angebot zum Selbstdatenschutz: $h t t p: / / w w w . d a t e n s c h u t z$. rlp.de/de/selbstds.php

\section{Kooperationsvereinbarung zur Internetsicherheit zwischen ENISA und europäischen Normungsgremien CEN und CENLEC}

Die Europäische Union (EU) Agentur ENISA unterstützt den Ausbau von Standards für Produkte und Dienstleistungen im Bereich Internetsicherheit durch die am 10.07.2013 erfolgte Unterzeichnung einer Kooperationsvereinbarung mit zwei der wichtigsten Normungsgremien in der EU, dem Europäischen Komitee für Normung (CEN) $)^{1}$ und dem Europäischen Komitee für elektrotechnische Normung (CENELEC) ${ }^{2}$. Der Kooperationsvertrag zielt darauf ab, einen effektiveren Beitrag zum Verständnis und zur Lösung von Netzund Informationssicherheitsthemen im Zusammenhang mit Standardisierung zu leisten, insbesondere in verschiedenen IKT-Sektoren, die von Bedeutung für ENISA sind. Dies steht im Einklang mit den neuen, erweiterten Aufgaben der kürzlich verabschiedeten ENISA Verordnung, die ENISA eher eine pro-aktive Rolle bei der Unterstützung der Entwicklung von NIS-Standards zuteilt.

Genauer umfasst die Vereinbarung:

- ENISAs Teilnahme als Beobachter in bestimmten Technischen Komitees, deren Arbeitsgruppen und Workshops von CEN und CENELEC, um bei der Ausarbeitung europäischer Standards zu unterstützen;

- CEN und CENELECs Auswertung relevanter ENISA Forschungsergebnisse und deren Übertragung auf Normungsarbeiten;

- ENISAs Teilnahme und gegebenenfalls den Vorsitz bei relevanten CEN und CENELEC Technischen Komitees, Arbeitsgruppen und Workshops in Übereinstimmung mit CEN-CENELEC internen Vorgaben;

\footnotetext{
1 http://www.cen.eu/cen/AboutUs/WhatisCEN/Pages/default.aspx

2 http://www.cenelec.eu/
}

- Die Verbreitung und Förderung von Informationen zu Publikationen, Ergebnissen, Tagungen, Seminaren;

- Die Bereitstellung gegenseitiger Unterstützung bei Werbeaktivitäten und dem Aufbau betrieblicher Kontakte sowie von Forschungsnetzwerken für netz- und informationssicherheitsnormenbezogene Standards bei den Aufgaben;

- Die Organisation thematischer Workshops, Konferenzen und Seminare zu Technologie- und Forschungsthemen mit Bezug auf Netz-und Informationssicherheitsnormungstätigkeiten;

- Der Austausch relevanter Informationen zu identifizierten Themen von allgemeinem Interesse.

\section{G\&D entwickelt KAMS für die zweite Generation der deutschen Gesundheitskarte}

Die AOK Systems und die Bitmarck Software, die beiden größten Systemhäuser der gesetzlichen Krankenkassen, haben am 08.08.2013 Giesecke \& Devrient (G\&D) mit der Weiterentwicklung des Kartenapplikations- und Management Systems (KAMS) für die zweite Generation der deutschen Gesundheitskarte beauftragt. Damit ist G\&D als Komplettanbieter nun für die Entwicklung des neuen Hintergrundsystems und der elektronischen Gesundheitskarte (eGK) verantwortlich: Bereits vor kurzem hatte die Gematik die Entwicklung für das Betriebssystem des Nachfolgers der heutigen elektronischen Gesundheitskarte an G\&D vergeben. Die nächste Generation der Gesundheitskarte soll ab Ende 2014 im Markt eingeführt werden.

Das neue Hintergrundsystem KAMS wird sowohl die neue, zweite Generation eGK als auch die bisherigen Karten verwalten können. Starttermin für das neue Hintergrundsystem ist ebenfalls Ende 2014. Zusätzlich beauftragen die AOK Systems und die Bitmarck Software G\&D mit der Wartung und den zukünftigen Anpassungen des KAMS bis zum Jahr 2021.

Von derzeit rund 69 Millionen Gesundheitskarten in Deutschland werden rund 60 Millionen über die erste Generation des Hintergrundsystems KAMS von G\&D verwaltet. Das KAMS von G\&D unterstützt die Krankenkassen bei der Herausgabe der Gesundheitskarten. Es steuert kartenindividuell die Kommunikation mit anderen Systemen und verwaltet, erneuert oder ergänzt sämtliche Daten, die auf den Gesundheitskarten gespeichert werden oder im Kartenausgabeprozess entstehen.

\section{SECUDIS: Sicherheitskomponenten für Chipkarten und Ausweise der Zukunft}

Die Bundesdruckerei GmbH, Bayer MaterialScience, die Fraunhofer-Institute IZM, IAP und EMFT sowie NXP Semiconductors haben das gemeinsame Forschungsprojekt "SECUDIS" nach drei Jahren erfolgreich abgeschlossen. Im Rahmen des Projekts wurden sicherheitstechnische Komponenten für Identitätsdokumente erforscht und entwickelt, die bei künftigen Produktentwicklungen zum Einsatz kommen sollen.

Die Abkürzung steht für "SECUre Digital Identity Solutions - Innovative Systeme für zukünftige Hochsicherheitsanwendungen". Drei Jahre lang haben die Projektpartner funktionelle und sicherheitstechnische Komponenten für benutzerfreundliche Ausweis- 